\title{
Using the whole plant to treat malaria
}

The development of drug resistance is a primary factor in the failure of malaria treatment strategies. For example, artemisinin is the drug most commonly used to treat human malaria worldwide, but some malaria parasites have developed resistance to it. Overcoming the development of resistance is therefore a key objective in the search for improved malaria treatments.

Researchers recently showed that treatment with the whole plant (Artemisia annua) from which artemisinin is derived could overcome existing resistance to artemisinin in a rodent malaria parasite. Moreover, the researchers estimated it would take at least three times longer for a second rodent malaria parasite to develop stable resistance to A. annua than to artemisinin. These data indicate that whole-plant therapy is more resilient to the development of resistance and therefore might have a longer effective 'lifespan' as a malaria treatment.

Stephen Rich (University of Massachusetts, Amherst) led the project to follow up on his group's earlier findings that $A$. annua was more effective than artemisinin in treating malaria in a rodent model. The new work specifically addressed resistance to $A$. annua versus artemisinin in two different rodent malaria parasites. "Conducting these experiments in different rodent malaria species...provides a robust test of the therapy," Rich explained in a press release. His group chose an artemisinin-resistant strain of Plasmodium yoelii to test whether A. annua could overcome existing drug resistance and chose Plasmodium chabaudi to track the development of resistance because it most closely resembles the deadliest human malaria parasite, Plasmodium falciparum.

In one set of experiments, mice were infected with an artemisinin-resistant strain of $P$. yoelii and then treated with high or low doses of A. annua or artemisinin. For each dose level, malaria parasitemia was lower in mice that received $A$. annua than in mice that received artemisinin (Proc. Natl. Acad. Sci. USA 112, 821-826; 2015). Next, Rich and colleagues used artificial selection experiments to monitor the development of resistance to A. annua or artemisinin in $P$. chabaudi under serial passage. Resistance to a low dose of artemisinin developed at passage 16 , at which point the artemisinin dose was doubled; resistance to the high dose developed after an additional

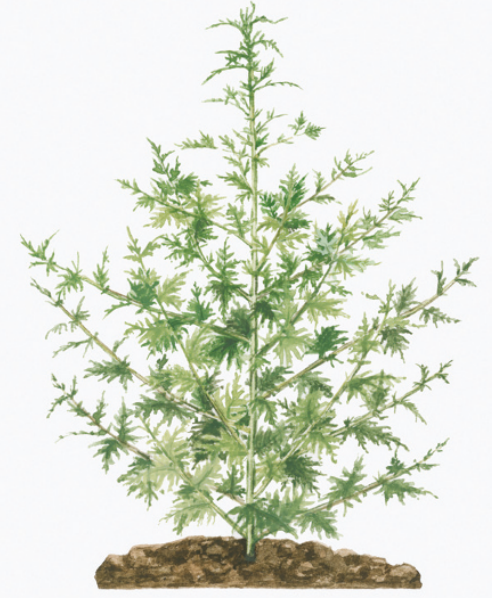

24 passages. In contrast, resistance to a low dose of $A$. annua did not develop even after 49 passages. The researchers conclude that the antimalarial activity and resilience of $A$. annua offer compelling reasons to pursue the use of A. annua or other nonpharmaceutical forms of artemisinin for the treatment of human malaria.

Monica Harrington

\section{A SPINAL NEURAL CIRCUIT KEEPS MICE MOVING}

Among many other jobs, the nervous system uses streams of information gathered from multiple senses to guide movement. The eyes detect obstacles. Balance gauges in the ears keep the head level. And sensors in the muscles and joints monitor limb position. Much of the resulting flood of information is preprocessed by sensory circuits before it reaches the brain. Visual signals, for instance, are processed by neurons and light sensors at the back of the eye before being transmitted to the visual centers in the brain. It seemed likely that touch signals were similarly processed by neural circuits in the spinal cord, but such circuits had not been identified-until recently. Neurobiologists at Salk Institute (La Jolla, CA) have now mapped the neural circuitry of the spinal cord that processes the sense of light touch by integrating motor commands from the brain with sensory signals from the limbs in mice.

The research group, headed by Martyn Goulding, traced the nerve fibers that carry signals from the touch sensors in the feet to their connections in the spinal cord in mice. These sensory fibers connected with a group of neurons expressing a receptor called ROR $\alpha$, which in turn connected with neurons in the motor region of brain. To investigate the role of ROR $\alpha$ neurons in touch and movement, the scientists selectively disabled these neurons in the spinal cords of mice. Mice lacking functional ROR $\alpha$ neurons were substantially less sensitive to light touch but were able to walk and stand normally on flat ground. When placed on a narrow, elevated beam, however, the mice had difficulty walking (Cell 160, 503-515; 2015). The scientists attributed this difficulty to an impairment of the ability to sense when a foot was slipping and respond with postural adjustment and correction of the foot position.

This study is part of a branch of research that aspires to explain how the nervous system processes and integrates sensory information to generate movement. "How the brain creates a sensory percept and turns it into an action is one of the central questions in neuroscience," stated Goulding in a press release. "0ur work is offering a really robust view of neural pathways and processes that underlie the control of movement and how the body senses its environment." A better understanding of these pathways could improve therapies for injuries and diseases that affect movement and balance.

Monica Harrington 